\title{
Biomaterials
}

\section{Electrochemical studies on the stability and corrosion resistance of titanium-based implant materials}

\author{
Maan Aziz-Kerrzo, Kenneth G. Conroy, Anna M. Fenelon, Sinead T. Farrell, \\ Carmel B. Breslin* \\ Department of Chemistry, National University of Ireland Maynooth, Maynooth, Co. Kildare, Ireland
}

Received 22 May 2000; accepted 28 September 2000

\begin{abstract}
The corrosion susceptibility of Ti, Ti-6Al-4V and Ti-45Ni was studied in a buffered saline solution using anodic polarisation and electrochemical impedance measurements. Pitting potentials as low as $+250 \mathrm{mV}$ (SCE) were recorded for Ti-45Ni and once initiated pits continued to propagate at potentials as low as $-150 \mathrm{mV}(\mathrm{SCE})$. It was possible to increase the pitting potential of $\mathrm{Ti}-45 \mathrm{Ni}$ to values greater than $+800 \mathrm{mV}(\mathrm{SCE})$ using a $\mathrm{H}_{2} \mathrm{O}_{2}$ surface treatment procedure; however, this surface modification process had no beneficial effect on the rate of pit repassivation. Impedance spectra, recorded under open-circuit conditions, were modelled using a dual oxide film model; a porous outer layer and an inner barrier oxide layer. The nature of this porous outer layer was found to depend on the nature of the electrode material and the presence of phosphate anions in the saline-buffered solution. The porous layers formed on Ti-45Ni and Ti-6Al-4V in the presence of phosphate anions had low resistances typically between 10 and $70 \Omega \mathrm{cm}^{2}$. Much higher porous layer resistances were recorded for $\mathrm{Ti}$ and also for $\mathrm{Ti}-45 \mathrm{Ni}$ and $\mathrm{Ti}-6 \mathrm{Al}-4 \mathrm{~V}$ in the absence of the phosphate anions. (C) 2001 Elsevier Science Ltd. All rights reserved.
\end{abstract}

Keywords: Implant; Corrosion; Pitting; Impedance spectroscopy

\section{Introduction}

Titanium and titanium-based alloys are employed widely in biomedical and dental applications. This is due, partly, to the stability and corrosion resistance exhibited by titanium, which in turn is due to the spontaneous formation of a passive titanium dioxide film, typically a few nm thick, which protects the metal from further oxidation [1]. It is commonly accepted that titanium exhibits a high stability and a high corrosion resistance in vitro [2,3], although there are reports, which show the accumulation of titanium in tissues adjacent to the implant $[4,5]$, signifying metal release and corrosion in vivo.

Titanium is alloyed frequently with other elements in biomedical applications. Some of the better known alloys include the Ti-6Al-4V and $\mathrm{Ti}-45 \mathrm{Ni}$ families, the latter are commonly termed shape-memory alloys. The shapememory effect arises because of a phase transition from

\footnotetext{
* Corresponding author.
}

the low-temperature martensite phase to the hightemperature austenite phase [6]. This phase transition enables the $\mathrm{Ti}-45 \mathrm{Ni}$ to recover completely from deformations even as high as $8 \%$ strain, and thus it is a very attractive material in certain biomaterial applications.

It is accepted that Ti-6Al-4V alloys exhibit high corrosion resistance $[3,7,8]$ under most conditions; however, there are a number of conflicting reports on the corrosion resistance of the shape-memory $\mathrm{Ti}-45 \mathrm{Ni}$ alloys [9-13]. In some cases, it has been reported that $\mathrm{Ti}-45 \mathrm{Ni}$ exhibits excellent resistance to corrosion while other researchers have reported the onset of pitting attack at relatively low potentials, which raises concern over the use of $\mathrm{Ti}-45 \mathrm{Ni}$ as an appropriate implant material.

In this communication, results are reported and discussed on the electrochemical behaviour and corrosion resistance of $\mathrm{Ti}, \mathrm{Ti}-45 \mathrm{Ni}$ and $\mathrm{Ti}-6 \mathrm{Al}-4 \mathrm{~V}$. Electrochemical techniques, both polarising and near-non-polarising impedance measurements were applied to study the corrosion susceptibility and stability of $\mathrm{Ti}-45 \mathrm{Ni}$ in a phosphate buffered saline solution. For comparative purposes, similar measurements were carried out on pure Ti and Ti-6Al-4V. 


\section{Experimental}

Electrodes were prepared from pure titanium (99.999\%), Ti-6Al-4V (Fe impurities <300 ppm) and Ti-45Ni (shape-memory alloy). The electrodes were provided in rod form and were embedded in epoxy resin in a Teflon holder with electrical contact being achieved by means of a copper wire threaded into the base of the metal sample. The $\mathrm{Ti}-45 \mathrm{Ni}$ wire electrodes were first polarised in $\mathrm{HNO}_{3}$-containing solution prior to electrode fabrication. It was found that this initial oxidation treatment coupled with careful electrode preparation eliminated the onset of crevice attack at the electrode-resin interface. Prior to each test, the exposed surfaces were polished to a smooth surface finish, using $\mathrm{H}_{2} \mathrm{O}$ lubricated, $1200 \mathrm{~g} \mathrm{SiC}$ paper, and rinsed with distilled water. High-density graphite rods were used as the auxiliary electrodes and a saturated calomel electrode (SCE) was used as the reference electrode, with all potentials represented relative to this electrode. The electrolyte consisted of a phosphate buffered-saline solution, $\mathrm{pH} 7.4$ $\left(8.77 \mathrm{~g} \mathrm{dm}^{-3} \mathrm{NaCl}, \quad 1.42 \mathrm{~g} \mathrm{dm}^{-3} \quad \mathrm{Na}_{2} \mathrm{HPO}_{4} \quad\right.$ and $2.72 \mathrm{~g} \mathrm{dm}^{-3} \mathrm{KH}_{2} \mathrm{PO}_{4}$ ). The electrolyte was maintained at a temperature of $37^{\circ} \mathrm{C}$ throughout all experiments.

Potentiodynamic electrochemical experiments were carried out using an EG \& G Potentiostat, Model 263. The working electrodes were polarised from below the corrosion potential $(30 \mathrm{mV})$ at a scan rate of $0.5 \mathrm{mV} \mathrm{s}^{-1}$ in the anodic direction, following an initial delay period of $30 \mathrm{~min}$ under open-circuit conditions. All current data are reported in terms of the geometric surface area. Impedance measurements were recorded using a Solartron 1250 Frequency response analyser and an EI 1287 electrochemical interface. The spectra were recorded at the corrosion potential following different immersion periods under open-circuit conditions. An excitation voltage of $10 \mathrm{mV}$ was used in all tests. All impedance data were fit to appropriate equivalent circuits using a complex nonlinear least-squares fitting routine, using both the real and imaginary components of the data.

\section{Results}

In Fig. 1, the anodic polarisation behaviours of pure $\mathrm{Ti}, \mathrm{Ti}-6 \mathrm{Al}-4 \mathrm{~V}$ and $\mathrm{Ti}-45 \mathrm{Ni}$ polarised in the phosphate buffer solution, $37^{\circ} \mathrm{C}$, at a scan rate of $0.5 \mathrm{mV} \mathrm{s}^{-1}$, are shown. The plots recorded for $\mathrm{Ti}-6 \mathrm{Al}-4 \mathrm{~V}$ and $\mathrm{Ti}$ are characteristic of highly passive systems. The passive current density was of the order of $2-3 \mu \mathrm{A} \mathrm{cm}^{-2}$ for the Ti electrode; this low passive current persisted even at potentials more anodic than $1600 \mathrm{mV}$. This passive current density is higher than that normally reported for titanium polarised in saline solutions, but this is connected with the higher scan rates used in this study compared to potentiostatic or slow scan rates of

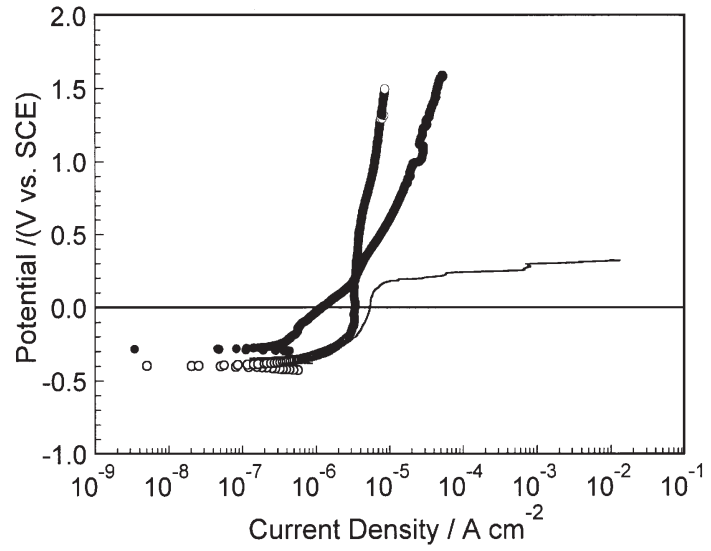

Fig. 1. Anodic polarisation plots recorded for $\bigcirc \mathrm{Ti}, \mathrm{Ti}-6 \mathrm{Al}-4 \mathrm{~V}$ and - Ti-45Ni in a $37^{\circ} \mathrm{C}$ phosphate buffer solution.

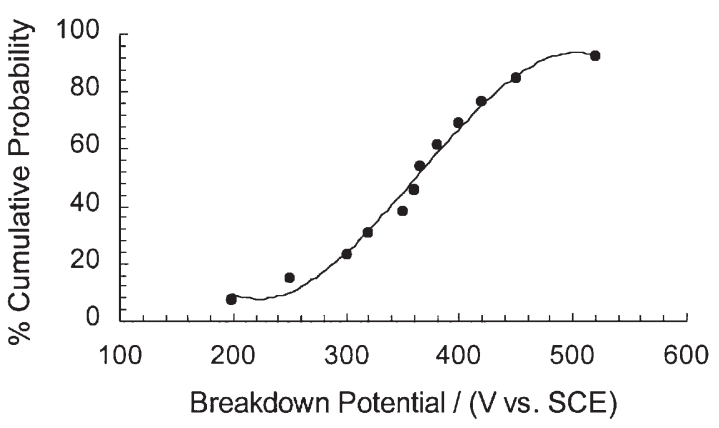

Fig. 2. Cumulative probability plot of the breakdown potentials recorded for $\mathrm{Ti}-45 \mathrm{Ni}$ in a $37^{\circ} \mathrm{C}$ phosphate buffer solution.

$0.167 \mathrm{mV} \mathrm{s}^{-1}$ normally employed. Similar behaviour was observed for the Ti-6Al-4V alloy, but the anodic current increased with increasing potential to reach values of approximately $20 \mu \mathrm{A} \mathrm{cm}^{-2}$ at $1.0 \mathrm{~V}$. However, the electrochemical behaviour of the shape-memory, Ti-45Ni alloy, is considerably different. A low passive current density, typical of a passive system, is observed at relatively low anodic potentials, but passive film breakdown and pitting attack, characterised by a sharp and steady increase in the anodic current, is observed in the potential region of $200 \mathrm{mV}$.

In order to investigate the statistical nature of the pitting attack observed for the $\mathrm{Ti}-45 \mathrm{Ni}$ electrodes, a number of pitting scans were carried out and the pitting potential calculated in terms of the cumulative probability of the pitting event. These data, shown with the pitting potentials presented as a function of the cumulative probability, are presented in Fig. 2. Here, it can be seen that the pitting potentials vary from the relatively low value of 200 to $530 \mathrm{mV}$, with an average value of $380 \mathrm{mV}$. These data show clearly that the Ti-45Ni electrode is susceptible to pitting attack. 


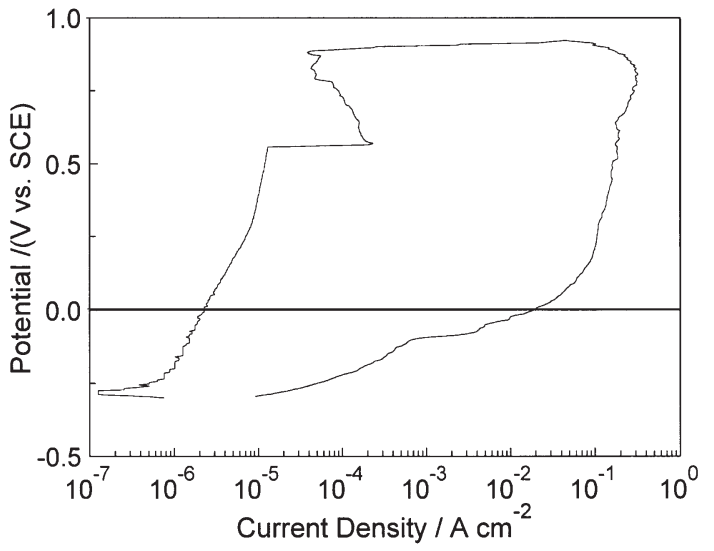

Fig. 3. Cyclic polarisation plot recorded for $\mathrm{Ti}-45 \mathrm{Ni}$ in a $37^{\circ} \mathrm{C}$ phosphate buffer solution.

Typical cyclic polarisation data recorded for $\mathrm{Ti}-45 \mathrm{Ni}$, shown in Fig. 3, which were recorded at a scan rate of $1.0 \mathrm{mV} \mathrm{s}^{-1}$ in the $37^{\circ} \mathrm{C}$ phosphate buffer solution, show that the Ti-45Ni electrode, once activated, is unlikely to repassivate. The forward scan, in which a low passive current density of approximately $1 \mu \mathrm{A} \mathrm{cm}^{-2}$ is measured, is consistent with passive behaviour. The sharp current increase observed at approximately $580 \mathrm{mV}$ marks the onset of pitting attack. This potential is somewhat higher than that observed in Figs. 1 and 2, but this is connected with the faster scan rate employed in these cyclic polarisation measurements. The current decreases temporarily on polarising the electrode further in the anodic direction, but then increases rapidly again at about $800 \mathrm{mV}$. The potential applied to the electrode was reversed and scanned in the electronegative direction once the anodic current increased beyond $1 \mathrm{~mA} \mathrm{~cm}^{-2}$. However, it can be seen from the plot that the anodic current continues to increase up to a current density of approximately $0.3 \mathrm{~A} \mathrm{~cm}^{-2}$ signifying increasing rates of pitting attack even though the potential applied to the electrode is lowered. This high anodic current is maintained even at $0 \mathrm{~V}$ indicating the complete lack of repassivation under these conditions.

It was possible to increase the resistance of the Ti-45Ni electrode to the onset, or initiation, of pitting attack using a surface modification procedure. Typical anodic polarisation plots recorded following modification of the Ti-45Ni electrode are shown in Fig. 4. The surface modification procedure involved polarising the electrode, from the open-circuit potential to $800 \mathrm{mV}$, or immersion in a $100 \mathrm{mM} \mathrm{H}_{2} \mathrm{O}_{2}$ solution with a supporting $0.1 \mathrm{M} \mathrm{Na}_{2} \mathrm{SO}_{4}$ electrolyte. Three separate plots, typical of the data obtained, are shown in the figure; these data show, clearly, that the surface modification procedure gives rise to a significant increase in the breakdown potential. However, these surface modification procedures did not influence the repassivation kinetics; once

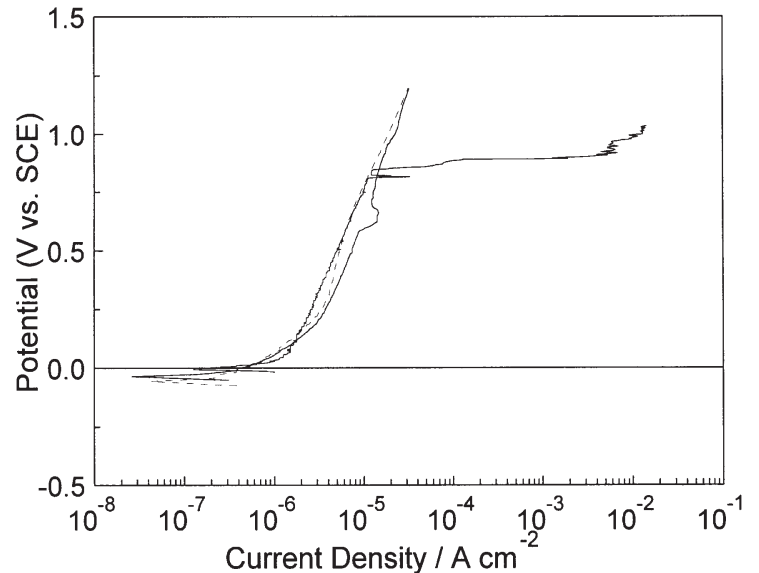

Fig. 4. Anodic polarisation plots recorded for $\mathrm{Ti}-45 \mathrm{Ni}$ in a $37^{\circ} \mathrm{C}$ phosphate buffer solution following modification in a $\mathrm{H}_{2} \mathrm{O}_{2}$-containing solution; -..- modified by polarising the electrode in the $\mathrm{H}_{2} \mathrm{O}_{2}$ solution, - modified by immersing the electrode in the $\mathrm{H}_{2} \mathrm{O}_{2}$ solution.

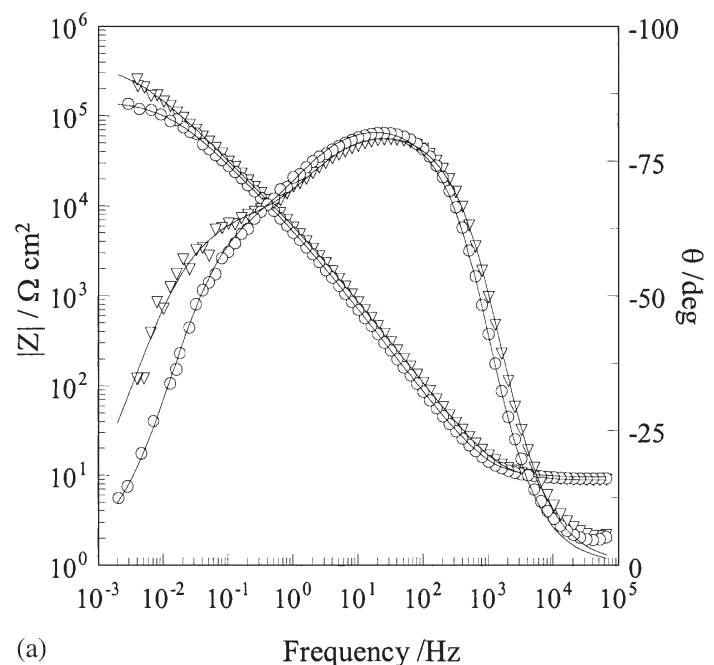

(b)

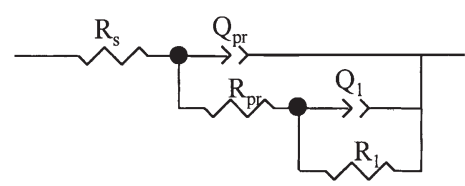

Fig. 5. (a) Impedance data recorded for $\mathrm{Ti}$ in a $37^{\circ} \mathrm{C}$ phosphate buffer solution; data recorded following $\bigcirc 300 \mathrm{~min}, \nabla 1000 \mathrm{~min}$, - simulated data, (b) equivalent circuit used in the generation of the simulated data.

activated the surface-modified electrodes continued to remain active at low potentials similar to that observed for the unmodified electrodes, Fig. 3.

In Fig. 5, typical electrochemical impedance data recorded for $\mathrm{Ti}$ in the $37^{\circ} \mathrm{C}$ phosphate buffer solution under open-circuit conditions, following 300 and 
1000 min immersion, are shown. In Fig. 5(a) the experimental data, shown by the symbols, and the simulated data, generated using the equivalent circuit depicted in Fig. 5(b), are shown. The equivalent circuit consists of resistance elements, $R$ and constant phase elements, $Q$. This equivalent circuit is based on a model used by Pan et al. [14] to simulate data for titanium in a saline solution. These authors viewed the surface layer formed on titanium as a two-layer oxide, with an inner barrier layer and an outer porous layer. Indeed, they and others [15] have shown, that under certain conditions, titanium supports a dense inner layer and a porous outer layer.

Using this two-layer oxide approach, $R_{\mathrm{s}}$ corresponds to the solution resistance, $R_{\mathrm{pr}}$ to the resistance of the outer porous layer, $R_{1}$ to the barrier layer resistance, $Q_{\mathrm{pr}}$ to the capacitance of the outer porous layer and $Q_{1}$ to the capacitance of the barrier layer. Although $Q_{\mathrm{pr}}$ and $Q_{1}$ are attributed to capacitance elements, constant phase elements were used as opposed to using pure capacitors in the fitting routine. Good agreement between the simulated and experimental data, as evident from a comparison of the experimental and simulated data in Fig. 5, was obtained when these constant phase elements were employed in the fitting routine. The impedance of a constant-phase element, is defined as $Z_{\mathrm{CPE}}=\left[Q(\mathrm{j} \omega)^{n}\right]^{-1}$ where $-1 \leqslant n \leqslant 1$ [16], with the value of $n$ being related to a non-uniform current distribution due to surface roughness or inhomogeneity. The $n$ value was approximately 0.75 in the case of $Q_{1}$ and in excess of 0.93 for $Q_{\mathrm{pr}}$ for all the fitted data. This shows that $Q_{\mathrm{pr}}$ is close to an ideal capacitor, but that $Q_{1}$ contains some frequency dispersion.

It can be seen from the impedance data presented in Fig. 5 that the system exhibits capacitive behaviour over a relatively wide-frequency region, which is typical of passive systems. On comparing the two sets of experimental data it is seen that the impedance response evolves with time. There is little difference in the profiles in the high- and medium-frequency region, but there exists a significant difference in the response at lower frequencies.

The evolution of the fitted parameters with time of immersion can be seen from the plots presented in Fig. 6. In Fig. 6(a), the resistance elements are shown as a function of time, while a similar plot in which the constant phase elements are shown as a function of time is presented in Fig. 6(b). The resistance element, $R_{\mathrm{pr}}$ remains essentially constant over the $900 \mathrm{~min}$ immersion period, but the element $R_{1}$, which represents the barrier layer resistance, increases with increasing immersion time. Likewise, in the plot depicted in Fig. 6(b), $Q 1$, which represents the capacitance of the barrier layer, decreases with immersion period, decreasing rapidly during the early period of immersion. Using the well-known parallel plate expression for the capacitance, $C$, to estimate the
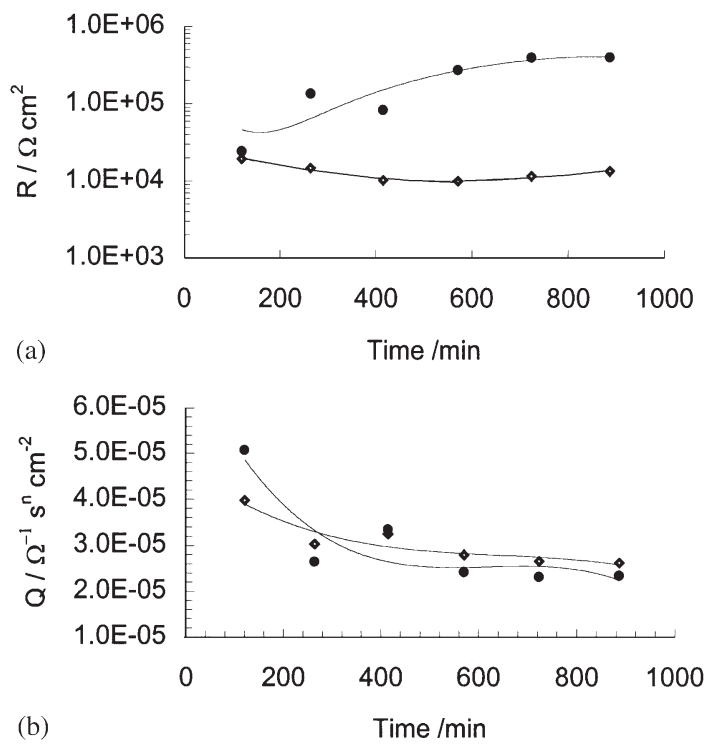

Fig. 6. (a) Resistance elements, $R_{1}, \diamond R_{\mathrm{pr}}$ and (b) constant phase elements, $Q_{1}$ and $\diamond Q_{\mathrm{pr}}$ plotted as a function of time for Ti in a $37^{\circ} \mathrm{C}$ phosphate buffer solution.

thickness of the passive layer,

$L=\frac{\varepsilon \varepsilon_{\mathrm{o}} A}{C}$

where $\varepsilon$ is the dielectric constant for the passive film, $\varepsilon_{\mathrm{o}}$ is the permittivity of free space and $A$ is the surface area, it can be seen that the decrease in $Q_{1}$ (which is equivalent to the capacitance $C$ ) is consistent with an increase in the thickness of the passive layer. The magnitude of the constant phase element, $Q_{\mathrm{pr}}$, which represents the outer porous layer, decreases slightly with continued immersion.

In Fig. 7(a), typical electrochemical impedance data recorded for Ti-6Al-4V in the $37^{\circ} \mathrm{C}$ phosphate buffered saline solution under open-circuit conditions, following 120 and 1000 min immersion, are shown. Here, as in the last case, the experimental data are shown by the symbols and the simulated data, which are generated using the equivalent circuit depicted in Fig. 7(b), are shown as the solid lines. Again, very good agreement between the simulated and experimental data is obtained. The equivalent circuit used in the fitting of these data was somewhat different to that already shown in Fig. 5(b), although the physical model of a dual oxide film was used. The equivalent circuit shown in Fig. 7(b) consists of a $R Q$ couple, labelled as $R_{\mathrm{pr}}$ and $Q_{\mathrm{pr}}$, which is used to model the outer porous layer while the barrier layer is modelled using the second grouping of $R_{1}, Q_{1}$ and $Q_{2}$. Here, $R_{1}$ refers to the resistance of the barrier layer, $Q_{1}$ is the capacitance of the barrier layer, with an $n$ exponent exceeding 0.9 , and $Q_{2}$, with an exponent of 0.5 , represents diffusion processes. 


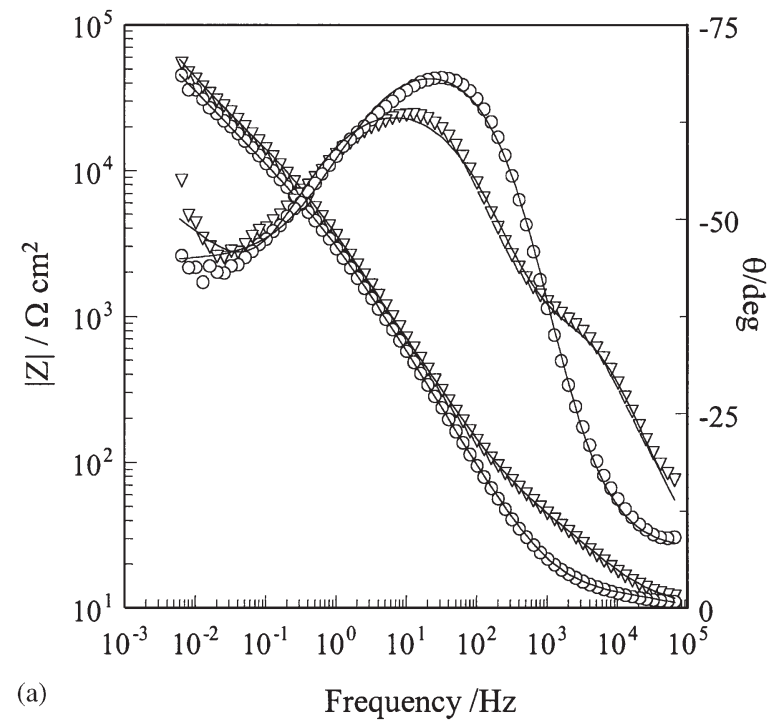

(b)
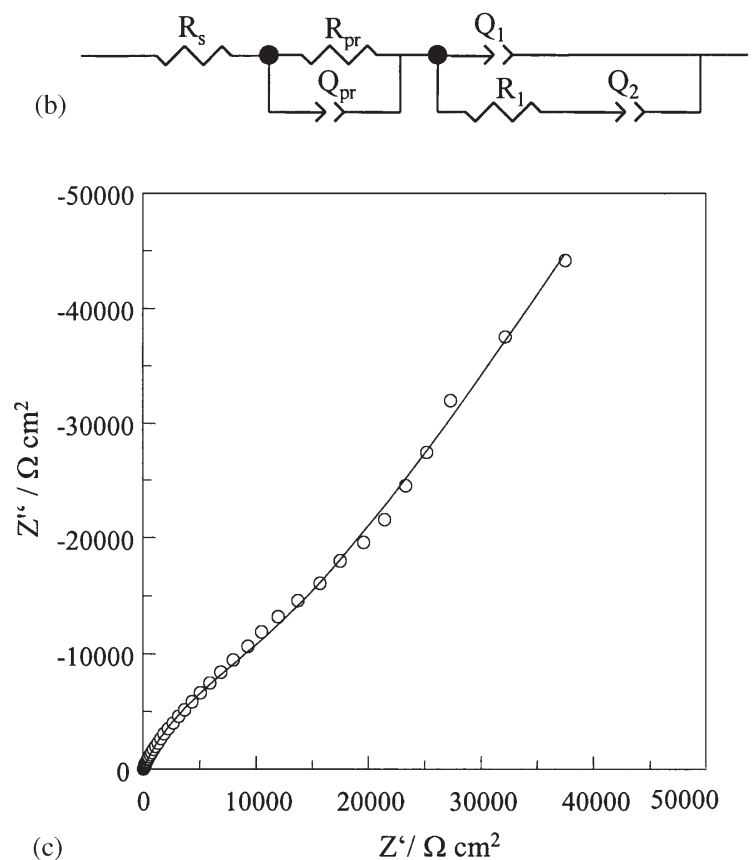

Fig. 7. (a) Impedance data recorded for Ti-6Al-4V in a $37^{\circ} \mathrm{C}$ phosphate buffer solution; data recorded following $\bigcirc 120 \mathrm{~min}, \nabla 1000 \mathrm{~min},-$ simulated data, (b) equivalent circuit used in the generation of the simulated data; (c) Nyquist plot recorded after $1000 \mathrm{~min}$.

The presence of a diffusion element can be seen more clearly from the data presented in Fig. 7(c), where the impedance data are presented on a Nyquist plot. The low-frequency data, which appears as a line drawn at an angle of $45^{\circ}$, is indicative of diffusion processes across the dual oxide layer on the implant electrode. It can be seen from the two plots shown in Fig. 7(a) that the nature of the Ti-6Al-4V passive layer varies with time. During the early stages of immersion, the impedance response is characterised by near-capacitive behaviour in the mid-
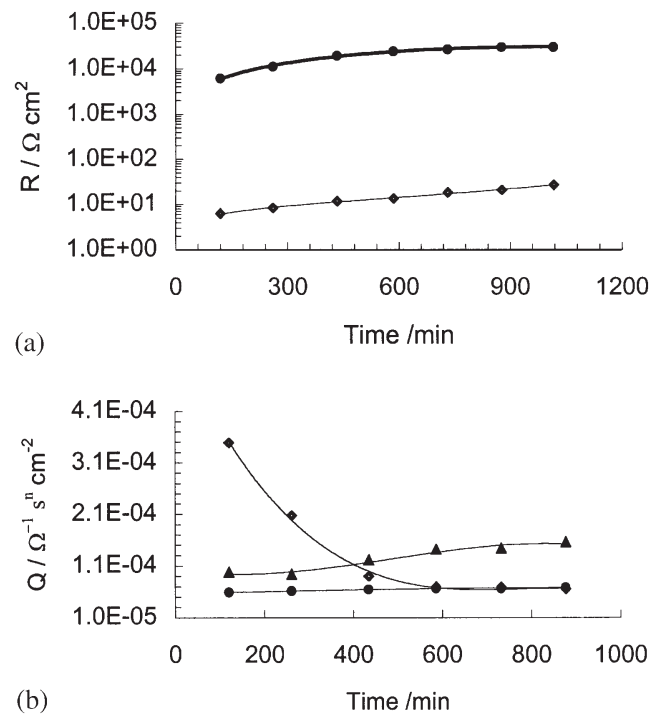

Fig. 8. (a) Resistance elements, $R_{1}, \diamond R_{\mathrm{pr}}$ and (b) constant phase elements, $Q_{1}, \diamond Q_{\mathrm{pr}}$ and $\boldsymbol{\Delta} Q_{2}$ plotted as a function of time for Ti-6Al-4V in a $37^{\circ} \mathrm{C}$ phosphate buffer solution.

frequency region, with diffusion processes evident at low frequencies. At this stage, the experimental data can be fit to either of the circuits depicted in Fig. 5(b) or 7(b). But, on continued immersion the emergence of a new time constant in the high-frequency region, $10^{4} \mathrm{~Hz}$, becomes evident, and at this stage the separate $R_{\mathrm{pr}} Q_{\mathrm{pr}}$ couple is required to model these data. This time constant at $10^{4} \mathrm{~Hz}$ is not connected with instrumental artefacts.

In Fig. 8(a) and (b), the resistance elements, $R_{\mathrm{pr}}$ and $R_{1}$, and the constant phase elements, $Q_{\mathrm{pr}}, Q_{1}$ and $Q_{2}$ are shown as a function of the immersion period for Ti-6Al-4V in the phosphate buffered saline solution. It is seen that $R_{\mathrm{pr}}$ is less than $5 \Omega \mathrm{cm}^{2}$ during the early stages of immersion increasing up to values typically of $30 \Omega \mathrm{cm}^{2}$. These values show that the porous layer formed has a very low resistance. In contrast, $R_{1}$, which represents the inner barrier layer resistance, is significant and remains at a high value throughout the measurement period. The constant phase element, $Q_{\mathrm{pr}}$, decreases significantly during the early period of immersion as the corresponding $R_{\mathrm{pr}}$ term increases. $Q_{1}$ remains essentially constant throughout the experiment indicating stability of the inner barrier passive film, while the diffusion component increases slightly with immersion time.

Similar impedance data recorded for $\mathrm{Ti}-45 \mathrm{Ni}$ in the phosphate buffered chloride solution are shown in Fig. 9. Again two plots, one recorded following $60 \mathrm{~min}$ and the other following $1000 \mathrm{~min}$ of immersion in the saline solution are shown, represented by the symbols, while the corresponding simulated data are shown by the solid lines. The equivalent circuit, shown in Fig. 7(b), was used to generate the simulated data. Again, good agreement between the experimental and simulated data is obtained. 


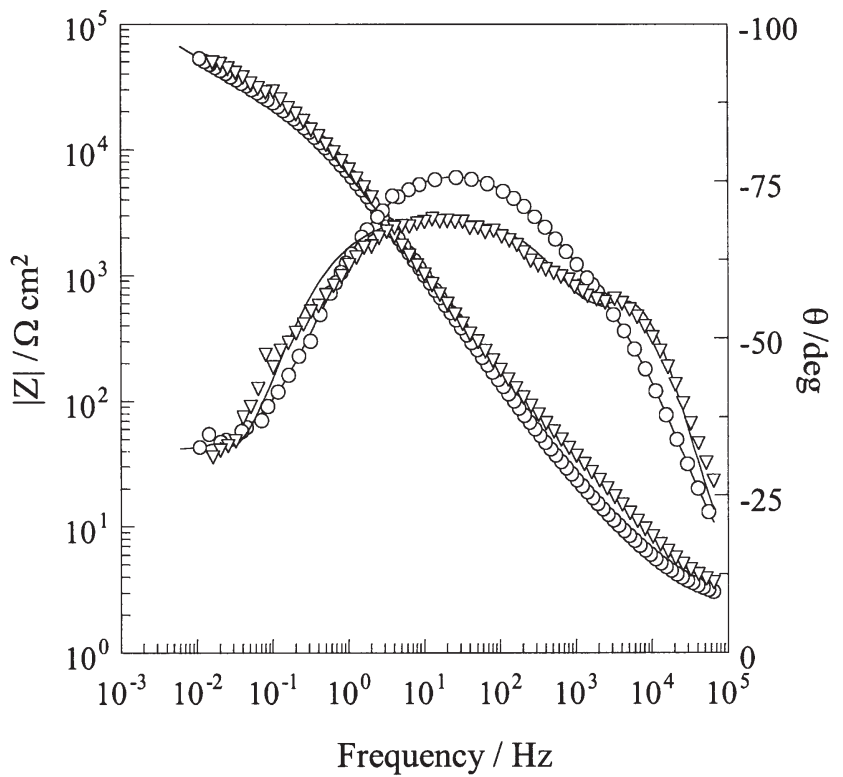

Fig. 9. Impedance data recorded for $\mathrm{Ti}-45 \mathrm{Ni}$ in a $37^{\circ} \mathrm{C}$ phosphate buffer solution; data recorded following $\bigcirc 60 \mathrm{~min}, \nabla 1000 \mathrm{~min}$, - simulated data.

These data are similar to those already presented for the Ti-6Al-4V system; the impedance response is typical of a near capacitive element that dominates the response in the medium-frequency region and a diffusion element that dominates the response in the low-frequency region, during the early stages of immersion. However, a highfrequency term is seen to develop following continued immersion.

The equivalent circuit parameters, $R_{\mathrm{pr}}$ and $R_{1}$, and the constant phase elements $Q_{\mathrm{pr}}, Q_{1}$ and $Q_{2}$ are shown for $\mathrm{Ti}-45 \mathrm{Ni}$ as a function of the immersion period in Fig. 10(a) and (b), respectively. Again, as seen for the Ti-6Al-4V system, $R_{\mathrm{pr}}$ increases initially from values less than $10 \Omega \mathrm{cm}^{2}$ to values of about $70 \Omega \mathrm{cm}^{2}$ on continued immersion, but again these values represent relatively low resistances. $R_{1}$ remains constant, adopting values typically of $4.0 \times 10^{4} \Omega \mathrm{cm}^{2}$ indicating stable passive conditions. $Q_{1}$, which represents the capacitance of the inner passive film, remains constant throughout the duration of the experiments. $Q_{\mathrm{pr}}$, which represents the outer porous layer decreases initially, but after short periods of time attains a steady-state value. Again, this reflects stability of the Ti-45Ni system, however there is evidence that the diffusion term, $Q_{2}$, increases slightly following immersion periods exceeding $2000 \mathrm{~min}$.

The main difference between the impedance data shown for $\mathrm{Ti}$, Ti-6Al-4V and $\mathrm{Ti}-45 \mathrm{Ni}$ is the time-dependence of the data and the fact that the data obtained for $\mathrm{Ti}-45 \mathrm{Ni}$ and $\mathrm{Ti}-6 \mathrm{Al}-4 \mathrm{~V}$ show the emergence of a time constant at high frequencies in the region of $1 \times 10^{4} \mathrm{~Hz}$. This can be associated with the formation of a porous layer on the electrodes, however, this layer
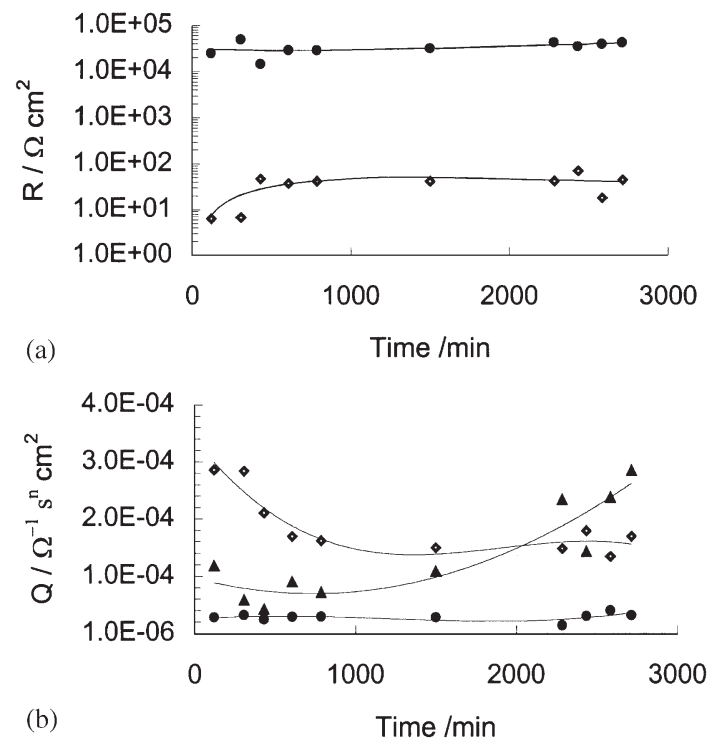

Fig. 10. (a) Resistance elements, $R_{1}, \diamond R_{\mathrm{pr}}$ and (b) constant phase elements, $Q_{1}, \diamond Q_{\mathrm{pr}}$ and $\boldsymbol{\Delta} Q_{2}$ plotted as a function of time for $\mathrm{Ti}-45 \mathrm{Ni}$ in a $37^{\circ} \mathrm{C}$ phosphate buffer solution.

appears to have different characteristics to that which exists on the pure titanium electrodes. In order to investigate the development of this layer further, and to determine what factors control its development, a series of measurements was performed in a $0.9 \% \mathrm{NaCl}$ solution, adjusted to a $\mathrm{pH}$ of 7.4. These data, which were obtained under open-circuit conditions and at $37^{\circ} \mathrm{C}$ are shown in Fig. 11(a) and (b) for Ti-6Al-4V and Ti-45Ni, respectively. Two profiles are shown on each plot; one recorded after $100 \mathrm{~min}$ of immersion and the other following $48 \mathrm{~h}$ of immersion, while simulated data, generated using the equivalent circuit depicted in Fig. 5(b) are shown as solid lines. The data obtained for Ti-6Al-4V show clearly that the impedance response remains constant and there is no evidence for the development of a distinct porous layer that dominates the impedance in the region of $10^{4} \mathrm{~Hz}$. The data shown for Ti-45Ni, Fig. 11(b), although showing evidence of two clear time constants, is clearly different in the high-frequency region to that shown in Fig. 9. It may be concluded from a comparison of the two sets of data obtained in the presence and absence of phosphate that the nature of the outer porous layer that forms on these electrodes, during the early stages of immersion, is dependent on the nature of the solution anions.

\section{Discussion}

The data presented in this communication show that $\mathrm{Ti}$ and $\mathrm{Ti}-6 \mathrm{Al}-4 \mathrm{~V}$ possess an apparently high resistance to the onset of localised corrosion under these experimental conditions, Fig. 1, and high stability under 


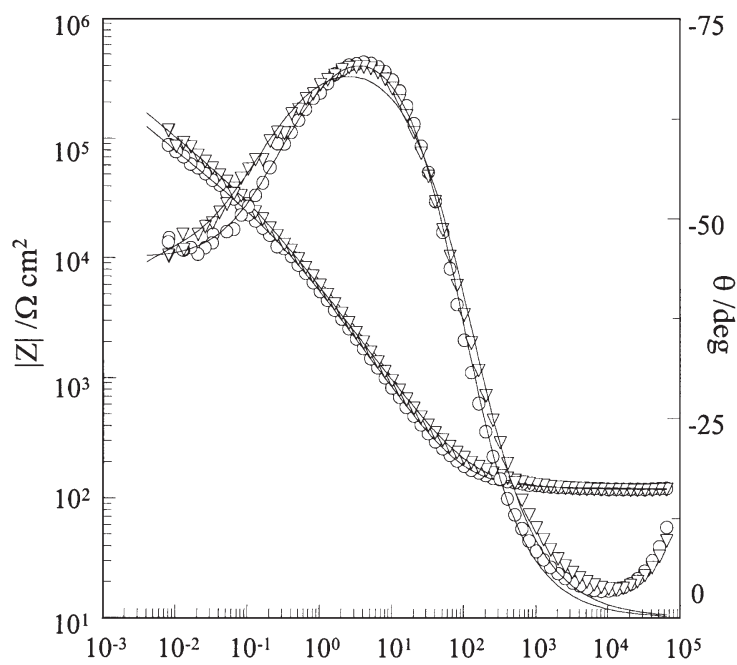

(a)

Frequency $/ \mathrm{Hz}$

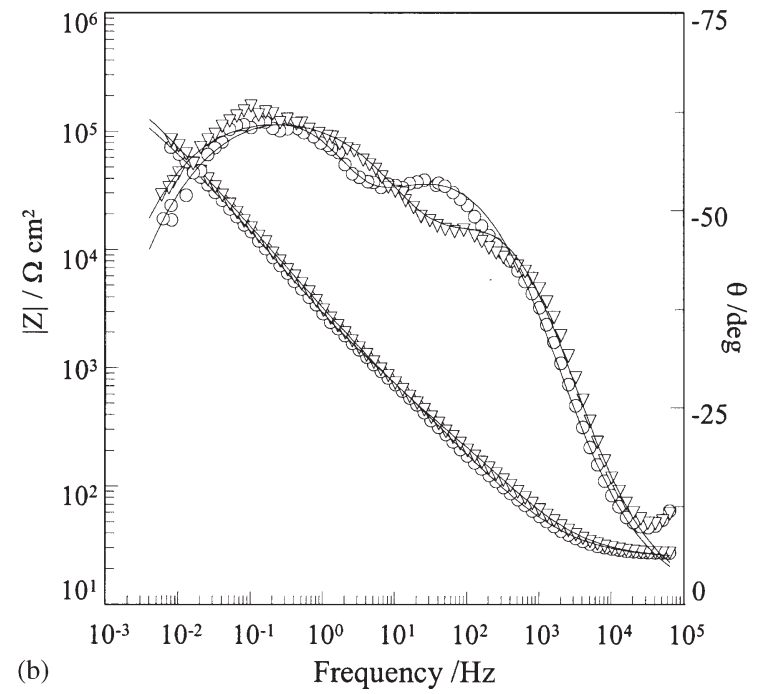

Fig. 11. Impedance data recorded in a $\mathrm{pH} 7.40 .9 \% \mathrm{NaCl}$ solution at $37^{\circ} \mathrm{C}$ following $\bigcirc 100 \mathrm{~min}$ immersion and $\nabla 48 \mathrm{~h}$ immersion periods --- simulated data, (a) data recorded for Ti-6Al-4V; (b) data recorded for $\mathrm{Ti}-45 \mathrm{Ni}$.

open-circuit conditions, Figs. 5-8, in agreement with several other reports $[1-3,7,8]$. However, the Ti-45Ni electrode is clearly susceptible to the onset of localised attack. Furthermore, there is a sizeable variation in the measured pitting potentials, Fig. 2, which is consistent with slight variations in the surface condition being connected with the pitting susceptibility. The open-circuit potential adopted by $\mathrm{Ti}-45 \mathrm{Ni}$ over extended immersion times in a $37^{\circ} \mathrm{C}$ phosphate buffered saline solution was typically $-120 \mathrm{mV}$. This value is only slightly removed from the lowest breakdown potentials measured (approximately $250 \mathrm{mV}$ ) which means that there exists a high probability of the potential adopted by the implant reaching values of the order of $250 \mathrm{mV}$ during long exposure conditions and localised attack initiating under these open-circuit conditions. Indeed, Hoar and Mears measured open-circuit potentials higher than $+250 \mathrm{mV}$ (SCE) for titanium alloys during in vivo experiments [17]. Furthermore, the equilibrium potential for the oxygen reduction reaction at a $\mathrm{pH}$ of 7.4 is $575 \mathrm{mV}$ (SCE). This potential is more noble than the lower pitting potentials measured, which means that the cathodic reduction reaction is feasible in the potential region where pitting is observed, thus, making the corrosion cell reaction spontaneous under non-polarising conditions. It is evident also from the data presented in Fig. 3 that once pits have initiated they propagate rapidly at much lower potentials and only repassivate when the electrode is polarised to potentials lower than the open-circuit potential adopted by $\mathrm{Ti}-45 \mathrm{Ni}$.

The surface modification data shown in Fig. 4 show that the pit initiation step is connected strongly with the nature of the surface and the passive film. The $\mathrm{H}_{2} \mathrm{O}_{2}$ in the surface modification solution acts as an oxidising agent, thus oxidising the passive layer on $\mathrm{Ti}-45 \mathrm{Ni}$. Indeed, this can be seen from a comparison of the anodic polarisation plots shown in Figs. 1 and 3 for the Ti-45Ni electrode; the $\mathrm{H}_{2} \mathrm{O}_{2}$ treatment gives rise to higher corrosion potentials, which is consistent with a reduction in the rate of the anodic half reaction. The pitting potentials measured following this treatment ranged from 800 to values in excess of $1200 \mathrm{mV}$, which are well above the potential region where the oxygen reduction reaction is feasible. Thus, in this case even though pits may initiate at these elevated potentials, the accompanying reduction reaction is not feasible and the corrosion event becomes non-spontaneous. Nevertheless, the slow rate of repassivation, which still persists for these surface-modified electrodes, remains a concern.

The electrochemical impedance data, which were recorded under open-circuit conditions, represent the response of the various electrodes to potential conditions that the electrodes are likely to adopt in vivo, but only during the early stages of immersion. The advantage of using this technique lies in the fact that it provides information on the corrosion resistance of the electrodes, and the nature of the electrode-solution interface, under nearly non-polarising conditions. The impedance data shown for pure $\mathrm{Ti}$ is characteristic of a stable system and is similar to that reported by Gonzalez et al. [3] and Pan et al. [14]. These data fit well to the dual oxide film model proposed by Pan et al. The data vary slightly with immersion time, the main variable being an increase in the resistance of the inner barrier layer, Fig. 6.

The impedance data recorded for the Ti-6Al-4V and the Ti-45Ni electrode, in the phosphate buffered solution are somewhat different. The impedance data show evidence for the formation of a porous outer layer, which dominates the impedance spectra in the region of $1 \times 10^{4} \mathrm{~Hz}$. It is evident from the data presented in 


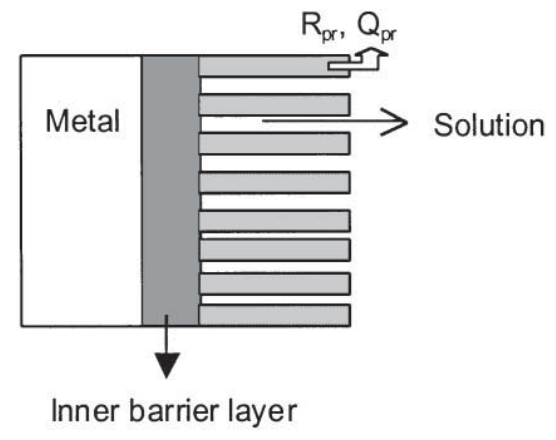

Fig. 12. Schematic representation of the porous-layer structure.

Figs. 8 and 10 that this porous layer has a very low resistance, ranging from 10 to about $70 \Omega \mathrm{cm}^{2}$. The constant phase element $Q_{\mathrm{pr}}$, which was used to model this porous layer, had exponent values that varied from 0.5 to 0.6 , indicating a diffusional process associated with this layer. This, coupled with the low resistance associated with this porous layer, points to a porous layer structure similar to that reported for anodised aluminium $[18,19]$, where the pores are filled with solution and $R_{\mathrm{pr}}$ corresponds to the resistance of the solution inside the pores. A schematic representation of this system is shown in Fig. 12. However, this scenario does not occur, to the same extent, in the absence of the phosphate species, as evident from a comparison of the impedance data recorded in the presence and absence of the phosphate species. The impedance data recorded in the absence of the phosphate species, Fig. 11, were fit to the equivalent circuit shown in Fig. 5, which is based on a two-phase oxide system, incorporating an outer porous oxide layer. In this case, the resistance associated with the porous layer was of the order of $800 \Omega \mathrm{cm}^{2}$, which is significantly higher than that measured in the presence of the phosphate species. In addition, the exponent of $Q_{\mathrm{pr}}$, was of the order of 0.8 , which is indicative of near-capacitive behaviour, which coupled with the higher $R_{\mathrm{pr}}$ resistance tends to suggest a more compact outer layer. However, the formation of this porous layer seems to have little effect on the pitting susceptibility of the electrodes, as there was no apparent change in the pitting potential of $\mathrm{Ti}-45 \mathrm{Ni}$ recorded as a function of the immersion period in the phosphate buffered saline solution.

\section{Conclusions}

The corrosion resistance of $\mathrm{Ti}$, Ti-6Al-4V and Ti-45Ni was studied in a saline-buffered solution. Ti-6Al-4V and Ti exhibited high resistances to the onset of localised corrosion, but pits were found to initiate at potentials as low as $250 \mathrm{mV}$ (SCE) on Ti-45Ni. More noble pitting potentials, exceeding $800 \mathrm{mV}$, were mea- sured for $\mathrm{Ti}-45 \mathrm{Ni}$ following a surface modification process in a $\mathrm{H}_{2} \mathrm{O}_{2}$-containing solution. However, repassivation potentials as low as $-150 \mathrm{mV}$ (SCE) were measured for the modified and unmodified Ti-45Ni electrodes, showing that once pits initiate they are capable of propagating at potentials significantly below the pitting potential. The passive films formed on these electrodes were analysed in terms of a dual oxide layer comprising an inner barrier and an outer porous layer. The nature of the porous layer was found to depend on the nature of the alloy and the solution anion species.

\section{Acknowledgements}

The authors gratefully acknowledge the support of this work by Enterprise Ireland, under the Basic Science Research Grants Award Scheme (Project Code SC/99/106).

\section{References}

[1] Knob LJ, Olson DL. Metals handbook. Corrosion, vol. 13, 9th edn. 1987. p. 669.

[2] Mueller HJ, Greener EH. Polarization studies of surgical materials in Ringer's solution. J Biomed Mater Res 1970;4:29.

[3] Gonzalez JEG, Mirza-Rosca JC. Study of the corrosion behaviour of titanium and some of its alloys for biomedical and dental implant applications. J Electroanal Chem 1999;471:109.

[4] Mu Y, Kobayashi T, Sumita M, Yamamoto A, Hanawa T. Metal ion release from titanium with active oxygen species generated by rat macrophages in vitro. J Biomed Mater Res 2000;49:283.

[5] Browne M, Gregson PJ. Effect of mechanical surface pretreatment on metal ion release. Biomaterials 2000;21:385.

[6] Tobushi H, Oshashi Y, Ohashi Y, Saida H, Shirai S. Recovery stress and recovery strain of TiNi shape memory alloy. JSME Int J I-Solid M 1992;35:84.

[7] Grosgogeat B, Reclaru L, Lissac M, Dalard F. Measurement and evaluation of galvanic corrosion between titanium/Ti-6Al-4V implants and dental alloys by electrochemical techniques and auger spectrometry. Biomaterials 1999;20:933.

[8] Lowenberg BF, Lugowski S, Chipman M, Davies DE. ASTMF86 passivation increases trace-element release from Ti-6Al-4V into culture-medium. J Mater Sci Mater Med 1994;5:467.

[9] Wever DJ, Veldhuizen AG, de Vries J, Busscher HJ, Uges DRA, van Horn JR. Electrochemical and surface characterization of a nickel-titanium alloy. Biomaterials 1998;19:761.

[10] Rondnelli G, Vincentini B, Cigada A. The corrosion behaviour of nickel titanium shape memory alloys. Corros Sci 1990;30:805.

[11] Fu Y, Wu XF, Wang Y, Li B, Yang SZ. Study of corrosion resistance property and microstructure of TiNi shape memory alloy modified by pulsed high-energy density plasma. Appl Surf Sci 2000;157:167.

[12] Rondelli G. Corrosion resistance tests on TiNi shape memory alloy. Biomaterials 1996;17:2003.

[13] Trepanier C, Tabrizian M, Yahia L, Bilodeau L, Piron DL. Effect of modification of oxide layer on NiTi stent corrosion resistance. J Biomed Mater Res 1998;43:433.

[14] Pan J, Thierry D, Leygraf C. Electrochemical impedance spectroscopy study of the passive oxide film on titanium for implant application. Electrochim Acta 1996;41:1143. 
[15] Tun Z, Noel JJ, Shoesmith DW. Electrochemical modification of the passive oxide layer on a Ti film observed by in situ neutron reflectometry. J Electrochem Soc 1999;146:988.

[16] Macdonald JR. Impedance spectroscopy. New York: Wiley, 1987

[17] Hoar TP, Mears DC. Corrosion resistant alloys in chloride solutions, materials for surgical implants. Proc R Soc A 1966;294:486.
[18] Mansfeld F, Zhang G, Chen C. Evaluation of sealing methods for anodized aluminum alloys with electrochemical impedance spectroscopy (EIS). Plat Surf Finish 1997;12:72.

[19] Mansfeld F, Chen C, Breslin CB, Hull D. Sealing of anodized aluminum alloys with rare earth metal salt solutions. J Electrochem Soc 1998;145:1715. 